\title{
Fractionalization in Josephson Junction Arrays Hinged by Quantum Spin Hall edges
}

\author{
Cenke $\mathrm{Xu}^{1}$ and Liang $\mathrm{Fu}^{1}$ \\ ${ }^{1}$ Department of Physics, Harvard University, Cambridge, MA 02138
}

(Dated: October 16, 2018)

\begin{abstract}
We study a novel superconductor-ferromagnet-superconductor (SC-FM-SC) Josephson array deposited on top of a two-dimensional quantum spin Hall insulator. The Majorana bound state at the interface between SC and FM leads to charge-e tunnelling between neighboring superconductor islands, in addition to the usual charge-2e Cooper pair tunnelling. Moreover, because Majorana fermions encode the information of charge number parity, an exact $Z_{2}$ gauge structure naturally emerges and leads to many new phases, including a deconfined phase where electrons fractionalize into charge- $e$ bosons and topological defects. A new SC-insulator transition has also been found.
\end{abstract}

PACS numbers:

\section{INTRODUCTION}

Superconductor Josephson junction arrays have been studied extensively in the past. At low temperature, the phase of a superconductor island becomes a quantum degree of freedom, which is conjugate to the Cooper pair density. Competition between charging energy and Josephson tunnelling leads to a superconductor-insulator transition at zero temperature, which is usually studied using quantum rotor models. Such a boson-only approach is no longer adequate if low-energy quasi-particles are present. Recently, it was proposed that if a $s$-wave superconductor (SC) and ferromagnet (FM) junction is hinged by the edge of a quantum spin Hall (QSH) insulator discovered recently ${ }^{1}$, a zero-energy Majorana bound state will be localized at the SC-FM interface ${ }^{2}$. Although the Majorana fermions do not carry the global $\mathrm{U}(1)$ charge of electrons, they do encode the information of fermion parity i.e. the even-odd of the electron number, hence Majorana fermion will participate in the tunnelling of charges between two SC islands, and enrich the physics of the Josephson array.

So far most work on topological insulators have been focusing on the band structure or weak interaction effects. Recently strong correlation effects for topological insulators have attracted more and more attentions ${ }^{4}-6$. Although it has been proposed that quantum computation may be realized based on Majorana bound states localized in topological $\operatorname{defects}^{3}$, very little was studied about the many-body or strong correlation effects that the Majorana fermions can participate in. In this work we will focus on the correlation physics in the Majorana fermion assisted Josephson array, and we will show that such Josephson arrays have unusual fractionalized phases and phase transitions.

\section{ONE DIMENSIONAL CASE}

Let us warm up with a simple one dimensional geometry, as depicted in Fig. 17a. We denote the location of every $\mathrm{SC}$ island by coordinate $j$, and denote the two
Majorana fermions around each $\mathrm{SC}$ island as $\gamma_{j, 1}$ and $\gamma_{j, 2}$. The FM islands have uniform magnetizations that are perpendicular to the angular momentum carried by the electrons of the QSH edge states, which opens up a gap for the QSH edge states. As was pointed out by Ref $\underline{7}$, these two Majorana fermions correlate with the fermion number on this $\mathrm{SC}$ island through the constraint $i \gamma_{j, 1} \gamma_{j, 2}=(-1)^{n_{j}}$. The tunnelling between SC islands has to be consistent with this constraint, and the following two terms are allowed 8 :

$$
\begin{aligned}
H_{t 1} & =\sum_{j}-t_{1} \cos \left(\phi_{j}-\phi_{j+x}\right) \\
H_{t 2} & =\sum_{j}-t_{2} i \gamma_{j, 2} \gamma_{j+x, 1} \cos \left[\left(\phi_{j}-\phi_{j+x}\right) / 2\right] .
\end{aligned}
$$

Here $\phi_{j}$ is the phase angle of the $\mathrm{SC}$ island $j$, and $e^{i \phi_{j}}$ increases the electron number $n_{j}$ by $2 . H_{t 1}$ is the ordinary Josephson tunnelling term, and $H_{t 2}$ is the charge- $e$ tunnelling assisted by the Majorana fermions, which is now allowed because with the Majorana fermions there is no longer a Cooper pair breaking gap between even and odd electron number on each $\mathrm{SC}$ island ${ }^{7} i . e$. electron can reside across the $\mathrm{SC}$ island nonlocally through majorana zero modes $\gamma_{j, 1}$ and $\gamma_{j, 2}$. Since $H_{t 1}$ is a second order effect that involves a Cooper pair breaking intermediate state, in the limit with dominant Cooper pair energy, $H_{t 1}$ is ignorable. Inclusion of small $H_{t 1}$ will only quantitatively change the physics discussed in this paper.

It is well-known that the one dimensional Majorana fermion is equivalent to a transverse field quantum Ising model, and the Ising variables are defined on the links of the 1d lattice denoted as $(j, j+x)$ in Fig. 1]

$$
\sigma_{j, j+x}^{x}=\prod_{k \leq j} i \gamma_{k, 1} \gamma_{k, 2}, \quad \sigma_{j, j+x}^{z}=i \gamma_{j, 2} \gamma_{j+x, 1}
$$

Now the full Hamiltonian can be written as

$$
H=\sum_{j} U\left(n_{j}-\bar{n}\right)^{2}-t_{2} \sigma_{j, j+x}^{z} \cos \left(\frac{\phi_{j}}{2}-\frac{\phi_{j+x}}{2}\right),
$$

which is subject to the constraint

$$
\sigma_{j-x, j}^{x} \sigma_{j, j+x}^{x}(-1)^{n_{j}}=1 .
$$




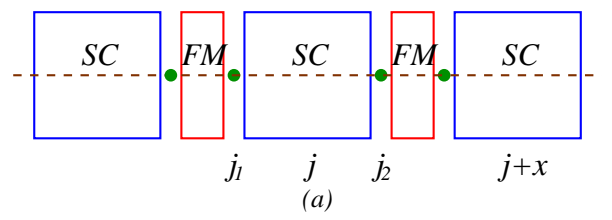

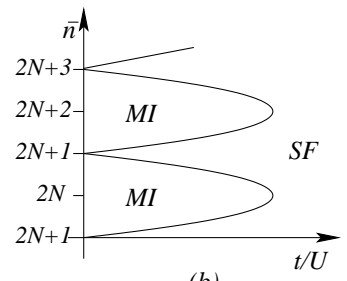

(b)

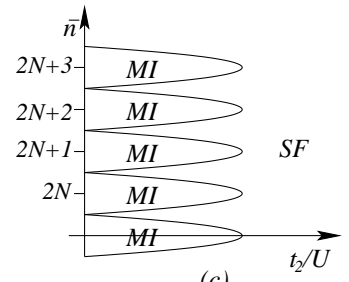

(c)
FIG. 1: $(a)$, the lattice structure for case 1. The green circles are the Majorana fermions, and the dashed brown line is the edge of the underlying QSH insulator. (b), the phase diagram for ordinary SC Josephson array. (c), the phase diagram for Josephson array in our case, where the number of MI lobes doubles compared with $(b)$.

The $U$ term represents a charging energy. The Hamiltonian Eq. 3 takes the standard form of a $Z_{2}$ gauge field $\sigma^{z}$ coupled to matter field $\phi / 2$. For this $1 \mathrm{D}$ system the $Z_{2}$ gauge field $\sigma_{j, j+x}^{z}$ can be absorbed into the rotor variable $\phi_{j}$ through the following duality mapping:

$\sigma_{j, j+x}^{z}=\tau_{j}^{z} \tau_{j+x}^{z}, \theta_{j}=\frac{\phi_{j}}{2}+\pi \frac{1-\tau_{j}^{z}}{2}, \sigma_{j, j-x}^{x} \sigma_{j, j+x}^{x}=\tau_{j}^{x}(5)$

$\tau_{j}^{z}, \tau_{j}^{x}= \pm 1$ are Ising operators defined on SC islands, and they satisfy the algebra of Pauli matrices. $\theta_{j}$ and $n_{j}$ satisfy the rotor phase-number algebra: $\left[\theta_{j}, n_{k}\right]=i \delta_{j k}$. Now this model can be written with the new variables as ordinary Bose-Hubbard mode ${ }^{9} \underline{\text { in }}$ 1D:

$$
H=\sum_{j} U\left(n_{j}-\bar{n}\right)^{2}-t_{2} \cos \left(\theta_{j}-\theta_{j+1}\right),
$$

and the gauge constraint operator $\sigma_{j, j-x}^{x} \sigma_{j, j+x}^{x}(-1)_{j}^{n}=$ $\tau_{j}^{x}(-1)^{n_{j}}$ commutes with $e^{i \theta_{j}}$.

The phase diagram of the model Eq. 3 is identical to model Eq. 6] with integer $\bar{n}$, there is a Mott-insulator (MI) phase with fixed fermion number on every SC island when $t_{2} / U \ll 1$, and a superfluid (SF) phase when $t_{2} / U \gg 1$. The phase diagram is shown in Fig. 1r. When $\bar{n}$ is precisely half-integer, since in this model there is no nearest neighbor density repulsion, the MI phase vanishes. Compared with the ordinary Josephson array, the number of MI lobes is doubled in our case (Fig. 1b, c), basically because there is no charge gap between even and odd particle filling. The MI phase is nondegenerate, every SC island has fixed charge number. In the SF phase, besides the Cooper pair operator $e^{i \phi}$, the following string operator also has algebraic correlation:

$$
\left\langle e^{i \phi_{j} / 2} \prod_{m=1}^{L} \sigma_{j, j+m}^{z} e^{-i \phi_{j+L} / 2}\right\rangle \sim\left\langle e^{i \theta_{j}} e^{-i \theta_{j+L}}\right\rangle \sim(1 / L)^{K} .(7)
$$

with the Luttinger parameter $K$ tuned by $t_{2} / U$.

Single electron can be injected into the system through the Majorana fermion bound state. One legitimate representation of electron operator is $c_{j, 1} \sim e^{i \phi_{j} / 2} \gamma_{j, 1} \underline{7,8}$. The spin index does not appear in the electron operator because the spin degeneracy is lifted by FM islands and the spin-orbital couplings in underlying QSH edge states. Also, under transformation $\phi_{j} \rightarrow \phi_{j}+2 \pi$, the solution of the Majorana bound state $\gamma_{j, a}$ changes sign, therefore the physical electron operator remains invariant. In terms of the bosonic variables, the electron operator can be expressed as

$$
c_{j, 1} \sim \prod_{k \leq j} \sigma_{k-x, k}^{z} \sigma_{j-x, j}^{x} e^{i \phi_{j} / 2} \sim \exp \left[i \pi \sum_{k<j} n_{k}\right] e^{i \theta_{j}} .
$$

The $\sigma^{x}$ in the product Eq. 8 guarantees the fermionic statistics between two electron operators. The correlation function between two electron operators is

$$
\left\langle c_{j-L, 1} c_{j, 1}^{\dagger}\right\rangle \sim\left\langle e^{i \theta_{j-L}} \exp \left[i \pi \sum_{k=j-L}^{L-1} n_{k}\right] e^{i \theta_{j}}\right\rangle \sim\left(\frac{1}{L}\right)^{K+\frac{1}{4 K}} \text {.(9) }
$$

The Bosonic representation of electron operator Eq. 8 takes exactly the same form as the standard fermionization of the Bose variables $\theta$ and $n$ in one dimension.

The phase transition at the integer filling is a Kosterlitz-Thouless (KT) transition at $K=1 / 4$, which physically corresponds to proliferating $2 \pi$ kinks of $\theta$ defined in Eq. 5, which is equivalent to a $4 \pi$ kink of $\phi$. At this transition, the scaling dimension of Cooper pair operator is $1 / 2$, while in ordinary 1D Josephson array, at the KT transition the Cooper pair operator has scaling dimension $1 / 8$, because normally the transition is driven by the proliferation of $2 \pi$ kink of $\phi$. The difference between these two cases stems from the charge- $e$ tunnelling enabled by the Majorana fermions. In terms of $\theta$ introduced in Eq. 5. $H_{t 1}$ is simply $\sum_{j}-t_{1} \cos \left(2 \theta_{j}-2 \theta_{j+x}\right)$, therefore turning on $H_{t 1}$ in Eq. 6] will not change the phase diagram, as long as there is no pairing gap between even and odd filling. The random one-dimensional lattice of Majorana fermions at the edge of a topological insulator, which is similarly connected to the random Ising model, has been discussed $\underline{10,11}$.

\section{TWO DIMENSION, REGULAR STRUCTURE}

Now let us move on to the 2D structure as depicted in Fig. 2 $a$. We place the SC and FM islands on top of a QSH insulator with commensurate holes. The internal edges of these holes hinge all the islands in this lattice. We first assume that the FM island is very thin compared with SC island, therefore the intra-island tunnelling between Majorana fermions is negligible compared with inter-island tunnellings. We denote every $\mathrm{SC}$ island as site $j$, and denote the four Majorana fermions around each SC island as $(j, a)$ with $a=1 \cdots 4$. The effective lattice is 
shown in Fig. 20 . Now the gauge constraint becomes $\gamma_{j, 1} \gamma_{j, 2} \gamma_{j, 3} \gamma_{j, 4}(-1)^{n_{j}}=1$. Again we can map the Majorana fermions to $Z_{2}$ gauge field as following:

$$
\begin{array}{r}
\sigma_{j, j+x}^{z}=i \gamma_{j, 2} \gamma_{j+x, 1}, \quad \sigma_{j, j+y}^{z}=i \gamma_{j, 3} \gamma_{j+y, 4} ; \\
\sigma_{j, j+y}^{x}=\prod_{k \leq j} i \gamma_{k, 4} \gamma_{k, 3}, \quad \sigma_{j, j+x}^{x}=\prod_{k \leq j} i \gamma_{k, 1} \gamma_{k, 2} .
\end{array}
$$

The entire Hamiltonian can be parallelly generalized from its $1 \mathrm{~d}$ counterpart Eq. 3

$$
H=\sum_{j} U\left(n_{j}-\bar{n}\right)^{2}-\sum_{\nu=x, y} t_{2} \sigma_{j, j+\nu}^{z} \cos \left(\frac{\phi_{j}}{2}-\frac{\phi_{j+\nu}}{2}\right)(11)
$$

Again this Hamiltonian is subject to the gauge constraint

$$
\sigma_{j, j-x}^{x} \sigma_{j, j+x}^{x} \sigma_{j, j-y}^{x} \sigma_{j, j+y}^{x}=(-1)^{n_{j}} .
$$

The $2 \mathrm{D} Z_{2}$ gauge field is drastically different from $1 \mathrm{D}$, in the sense that it has a nontrivial liquid phase even when $\phi_{j}$ is disordered. In the MI phase of SC islands, integrating out the gapped fluctuation $\phi_{j}$ will induce gauge invariant dynamics for $Z_{2}$ gauge field:

$$
H_{\text {ring }}=\sum_{j}-K \sigma_{j, j+x}^{z} \sigma_{j+x, j+x+y}^{z} \sigma_{j+y, j+x+y}^{z} \sigma_{j, j+y}^{z},
$$

with $K \sim t_{2}^{4} / U^{3}$. This is a standard ring exchange term of $Z_{2}$ gauge field. This term favors the ring product of $\sigma^{z}$ around each unit plaquette to be 1 . In ordinary $Z_{2}$ gauge field, this ring exchange term will compete with the $Z_{2}$ string tension term $\sum_{i, \nu}-h \sigma_{i, i+\nu}^{x}$, and when $K$ dominates $h$ the system is in a $Z_{2}$ liquid phase with topological order which cannot be described by local order parameter $\frac{12}{2}$. When $h$ dominates $K$, the system is in a confined phase without topological order, where $Z_{2}$ charged matter is not just gapped, but also confined spatially by a linear potential. Physically these two phases can be understood by the behavior of "vison", which is a topological excitation with the product $\prod_{\square} \sigma^{z}=-1$ on one plaquette. The vison carries a global $Z_{2}$ charge, because one can only create/anihilate a pair of vison by operator $\sigma_{i, i+\nu}^{x}$. In the liquid phase, the vison number is conserved mod 2, while in the confined phase the global $Z_{2}$ symmetry is spontaneously broken. This effect is manifested in the dual description of the $Z_{2}$ gauge field, which is formulated through the mapping: $\prod_{\square} \sigma^{z}=\tau_{\bar{j}}^{x}$, $\sigma_{j, j+y}^{x}=\tau_{\bar{j}-x}^{z} \tau_{\bar{j}}^{z} . \bar{j}$ denotes the dual lattice shown in Fig. 2 2 . Therefore the ordinary quantum $Z_{2}$ gauge field is dual to a $2 \mathrm{~d}$ transverse field quantum Ising model $H=\sum_{\bar{j}, \nu}-h \tau_{\bar{j}}^{z} \tau_{\bar{j}+\nu}^{z}-K \tau_{\bar{j}}^{x}$. When $K \gg h$, this Ising model is in the disordered phase, where the $Z_{2}$ conservation of $\tau^{x}$ (vison number) is preserved; when $h \gg K$, the $Z_{2}$ global symmetry of $\tau^{z}$ is spontaneously broken.

In our case, operator $\sigma_{j, j+\nu}^{x}$ is a nonlocal product of Majorana fermions, hence the string tension term $\sigma_{j, j+\nu}^{x}$ cannot exist in the Hamiltonian. Therefore in the disordered phase of $\phi_{j}$ (MI of SC islands), the local vison number commutes with the Hamiltonian i.e. vison

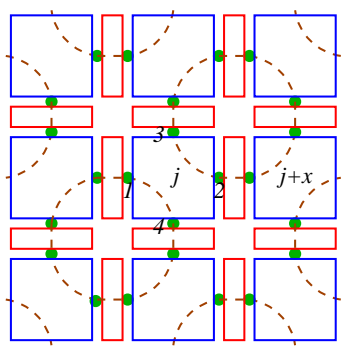

(a)

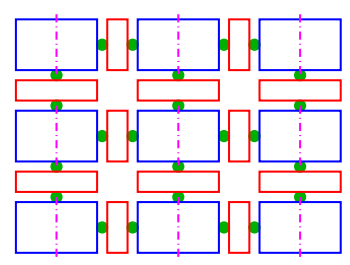

(c)

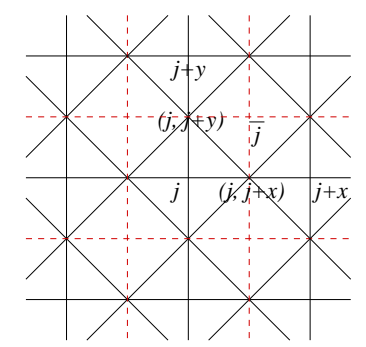

(b)

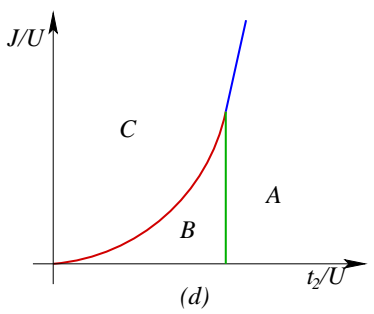

FIG. 2: (a), the Josephoson lattice structure of case 2. (b), the effective lattice of the Josephson array in $(a)$. The SC islands are denoted by $j$, and the $Z_{2}$ gauge field $\sigma^{x}$ and $\sigma^{z}$ are defined on links $(j, j+\nu)$. The dual Ising variables $\tau^{a}$ are defined on the sites $\bar{j}$ of the dual lattice, represented by red dashed lines. $(c)$, case 3 with compressed $y$ direction, the intra-island tunnelling $J i \gamma_{j, 3} \gamma_{j, 4}$ is denoted by the pink dashed lines. $(d)$, the phase diagram of case 3 with integer $\bar{n}$, plotted against $t_{2} / U$ and $J / U$. Phase $A, B$ and $C$ represent the SF, the $Z_{2}$ liquid, and the $\sigma^{x}$ ordered phase respectively. The green line between phase $A$ and $B$ is a $3 d \mathrm{XY}$ transition, and the transition between $B$ and $C$ is a first order transition described by Eq. 18 The direct transition between phase $A$ and $C$ may expand into a stable roton liquid phase.

is completely static. Hence the $Z_{2}$ gauge field is in its liquid phase. The $Z_{2}$ liquid phase is deconfined i.e. an extra electron will carry an infinite string of $\sigma^{x}$ due to the gauge constraint, but the energy cost is finite. For instance, the electron operator at site $j, 1$ (Fig. 2 $2 a$ ) can be written as

$$
c_{j, 1} \sim e^{i \phi_{j} / 2} \gamma_{j, 1} \sim \prod_{k \leq j} \sigma_{k-x, k}^{z} \sigma_{j-x, j}^{x} e^{i \phi_{j} / 2},
$$

where the product includes all the $\sigma^{z}$ on $x$-links to the left or below site $j$ (Fig. 3 $a$ ). $\sigma_{j, j+\nu}^{x}$ creates a pair of vison excitations, and since in the $Z_{2}$ liquid state the vison excitation is gapped and conserved, the least energy consuming behavior of an injected electron is to form a bound state with two visons, and become a charge-e boson represented by following operator:

$$
b_{j} \sim \prod_{k \leq j} \sigma_{k-x, k}^{z} \exp \left(i \phi_{j} / 2\right) .
$$

The SF phase can also be viewed as a condensate of $b_{j}$. If an electron is injected into this system, it will fractionalize into a mobile charge-e boson and a pair of static visons. If a Cooper pair is injected into this system, it will fractionalize into two bosons, instead of two electrons. 


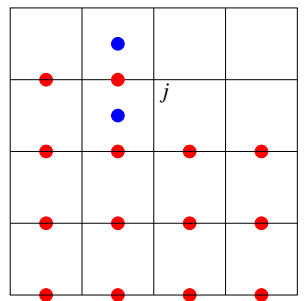

(a)

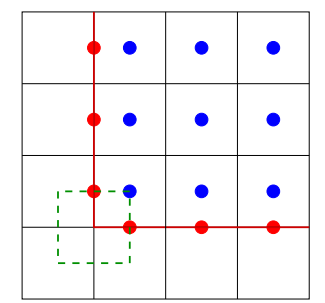

(b)
FIG. 3: (a), the electron at site $j, 1$ (Fig. 2 $a$ ) can be represented as $\exp \left(i \phi_{j} / 2\right)$ times a string of $\sigma^{z}$, denoted by red circles; By binding two visons (blue circles), the fermion becomes a boson. (b), the Wilson loop operator as a product of $\sigma^{z}$ (red circles), in the dual formalism is a product of $\tau^{x}$ on the dual lattice (blue circles). In the $Z_{2}$ liquid phase, the perturbation of $J$ term will only change the Wilson loop at the corner, represented by the green dashed square.

In this liquid phase, the boson and the vison will have mutual semion statistics i.e. when a boson $b_{j}$ encircles a vison through a close loop, the system wave-function acquires a minus $\operatorname{sign}^{12}$.

The SF phase has vortex with $h c / 2 e$ magnetic flux, which is bound with a vison. If we start from the SF phase, the $Z_{2}$ liquid phase can be viewed as a condensate of the double vortex with $h c / e$ magnetic flux of SF phase, since the MI liquid phase still has vison conservation, and the product of quantum circulation of the vortex and the charge in the vortex condensate is a constant: $Q_{v} Q_{e}=h a^{13}$. The $Z_{2}$ gauge field has no gapless photon excitations, hence at the transition between $\mathrm{SF}$ phase and the $Z_{2}$ liquid phase, the $Z_{2}$ gauge field does not introduce further anomalous dimension to $\psi \sim \exp (i \phi / 2)$. For instance, when $\bar{n}$ is integer this transition is a $3 \mathrm{~d}$ $\mathrm{XY}$ transition described by order parameter $\psi$. However, the physical Cooper pair operator $\psi^{2} \sim \exp (i \phi)$ gains a rather large anomalous dimension, which has been calculated by various methods $14: \eta_{\psi^{2}} \sim 1$.47. Similar situations were discussed at the transition between $Z_{2}$ spin liquid and spiral order in frustrated magnets 15,16 .

There is another equivalent way of describing the fractionalized $Z_{2}$ liquid phase i.e. the disordered phase of $\phi$. Let us assume the filling on every SC island is even, and we can define Ising variables

$$
\begin{aligned}
& \mu_{j}^{x}=i \gamma_{j, 1} \gamma_{j, 3}=i \gamma_{j, 2} \gamma_{j, 4}, \\
& \mu_{j}^{y}=i \gamma_{j, 3} \gamma_{j, 2}=i \gamma_{j, 1} \gamma_{j, 4}, \\
& \mu_{j}^{z}=i \gamma_{j, 1} \gamma_{j, 2}=i \gamma_{j, 4} \gamma_{j, 3} .
\end{aligned}
$$

$\mu^{a}$ satisfy the algebra of Pauli matrices. Notice that unlike $\sigma^{a}$ introduced before, now the Ising variables $\mu^{a}$ are defined on the sites of the lattice instead of the links. Now the ring exchange term $H_{\text {ring }}$ reads

$$
H_{\text {ring }}=\sum_{j}-K \mu_{j}^{x} \mu_{j+x}^{y} \mu_{j+y}^{x} \mu_{j+x+y}^{y} .
$$

This is precisely the model introduce in Ref $\frac{17}{7}$, as a concrete example with topological orders. This model is equivalent to the toric code model ${ }^{12}$, which becomes manifest once we switch the definition of $\mu^{x}$ and $\mu^{y}$ in Eq. 16 for one of the two sublattices of the square lattice.

\section{TWO DIMENSION WITH INTRA-ISLAND TUNNELING}

Now we consider a lattice structure slightly different from the previous section, with the entire system compressed in the $y$ direction, until there is a considerable intra-island tunnelling $-J i \gamma_{j, 3} \gamma_{j, 4}$. Using the mapping derived in last section, this operator is $J \sigma_{j, j-y}^{x} \sigma_{j, j+y}^{x}$ in terms of the $Z_{2}$ gauge variables, and this term becomes a ring exchange on the dual lattice $\sigma_{j, j-y}^{x} \sigma_{j, j+y}^{x}=$ $\tau_{\bar{j}}^{z} \tau_{\bar{j}-x}^{z} \tau_{\bar{j}-x-y}^{z} \tau_{\bar{j}-y}^{z}$, which represents a pair hopping of visons. Notice that $\tau^{z}$ are defined on the sites of the dual lattice instead of the links. The Hamiltonian of the dual Ising variables in the MI phase reads

$$
H=\sum_{\bar{j}}-J \tau_{\bar{j}}^{z} \tau_{\bar{j}-x}^{z} \tau_{\bar{j}-x-y}^{z} \tau_{\bar{j}-y}^{z}-K \tau_{\bar{j}}^{x} .
$$

This is precisely the model studied in Ref $\frac{18}{10}$ in the context of $p \pm i p$ superconductor Josephson array. The symmetry of this model is quasi-local in the sense that we can reverse the sign of $\tau^{z}$ along any column or row arbitrarily, without changing the Hamiltonian. Physically this symmetry means that the vison number has $Z_{2}$ conservation along each row and column on the dual lattice.

The phase diagram of model Eq. 18 has been studied both analytically and numerically $18-20$. Because of its special self-duality structure, it is expected that a transition occurs at precisely $J=K \sim t_{2}^{4} / U^{318,21}$. When $K>J$, the system is in the $Z_{2}$ liquid phase with topological order; when $J>K$, the topological order vanishes and the ground state is nondegenerate, although formally the nonlocal operator $\sigma^{x}$ has nonzero expectation value. Due to the absence of string tension $-h \sigma_{j, j+\nu}^{x}$, $Z_{2}$ charged matter is deconfined in both phases, which is very different from the ordinary $Z_{2}$ gauge field. Mean field argument as well as numerical results suggest that the transition at $J=K$ is first order $\underline{19}, 20,22$. In the $Z_{2}$ liquid phase, the $J$ term enables the visons to move in pairs, therefore an injected electron will fractionalize into two mobile parts: charge- $e$ boson and vison pair. Notice that unlike the ordinary $Z_{2}$ gauge field, this pair of visons cannot annihilate each other, due to the quasi local conservation of vison numbers discussed in last paragraph.

The difference between these two phases can be further characterized by the Wilson loop. In the phase with $J>$ $K$, since the zeroth order ground state with $K=0$ is an eigenstate of $\tau^{z}$, the Wilson loop $\left\langle\prod_{\mathcal{C}} \sigma^{z}\right\rangle=\prod_{\mathcal{A}} \tau^{x}$ can be calculated perturbatively with expansion of $K$, and it falls off according to an area law: $\left\langle\prod_{\mathcal{C}} \sigma^{z}\right\rangle \sim(K / J)^{\mathcal{A} 23}$. Here $\mathcal{C}$ and $\mathcal{A}$ represent a closed loop and the area enclosed inside this loop respectively. In the $Z_{2}$ liquid phase 
with $K>J$, it is usually expected that with the presence of transverse field $\sum_{j}-h \sigma_{j, j+\nu}^{x}$ the Wilson loop falls off with a perimeter law, which can also be revealed with a perturbation of $J / K$ on the ground state with $J=0$, where the Wilson loop is a constant. However, In our situation with the intra-island tunnelling, the first order expansion of $J$ term in Eq. 18 will only change the Wilson loop at the corners of loop $\mathcal{C}$ (Fig. $3 \mathrm{~b})$. Therefore we expect the Wilson loop in the $Z_{2}$ liquid phase falls off as a special "corner law" $\left\langle\prod_{\mathcal{C}} \sigma^{z}\right\rangle \sim e^{-\mathcal{N} J / K}, \mathcal{N}$ is the number of corners of this Wilson loop $\mathcal{C}$.

Based on the analysis above, when $J$ is small, by reducing $t_{2} / U$ from infinity we will first drive a transition from the SF phase to a $Z_{2}$ liquid phase, and then enters an "area law" MI phase through a first order transition. When $J$ is large enough, there can be a direct transition between the SF phase and the "area law" phase. This transition can be viewed as proliferating the $h c / 2 e$ vortices of the SF phase which can only move in pairs due to the quasi-local conservation of visons. This type of paired directional vortex dynamics was the key of the roton liquid phase proposed before $\mathrm{e}^{21,24,25}$, which is a stable phase with gapless vison excitations and quasi one dimensional dispersions. Therefore the direct transition in Fig. 2 $d$ might expand into a stable roton liquid phase. We will leave this possibility to future studies 26 .

If we turn on not only $-J_{j}^{z} i \gamma_{j, 3} \gamma_{j, 4}$, but also $-J_{j}^{x} i \gamma_{j, 2} \gamma_{j, 3}$ and $-J_{j}^{y} i \gamma_{j, 3} \gamma_{j, 1}$, after introducing Ising variables $\mu^{a}$ as Eq. 16. the model describing the system becomes

$$
\begin{gathered}
H=\sum_{j}-K \mu_{j}^{x} \mu_{j+x}^{y} \mu_{j+y}^{x} \mu_{j+x+y}^{y} \\
+J_{j}^{x} \mu_{j}^{x}+J_{j}^{y} \mu_{j}^{y}+J_{j}^{z} \mu_{j}^{z} .
\end{gathered}
$$

It will be interesting to do a full analysis of all the possible phases of this model with different choices of site dependent transverse fields $J_{j}^{a}$. For instance, with $J^{x}=J^{y}=0$, and $J^{z}$ is site independent, this model reduces to the model in Ref $\underline{18}$. If $J_{A}^{x}=J_{B}^{y} \neq 0(A$ and $B$ are two different sublattices of the square lattice), while all the other transverse fields are zero, this model is equivalent to the toric code model with one component of transverse magnetic field, and there is a confine-deconfine phase transition driven by this transverse field.

\section{SUMMARY}

In summary, Josephson array with Majorana fermion zero modes around each SC island is described by a precise $Z_{2}$ gauge field and matter field formalism, which leads to unusual fractionalization features in both one and two dimensions. Various pseudo-spin models with topological phases can be realized with the Josephson array. The fractionalization can be measured with single electron tunnelling experiments, because an electron will fractionalize into a boson and topological defects, and the single electron green's function becomes a convolution of two fractional excitations. This will be discussed in more details in another paper. In our current paper we focus on the disordered phase of the Josephson array with fractional excitations, but when $H_{t 1}$ is nonzero or $\bar{n}$ is away from integer, many interesting phase transitions can occur inside the SF phase, we will also study these physics in future ${ }^{26}$.

The authors are sponsored by the Society of Fellows, Harvard University, and the Milton Funds.
1 M. Konig, S. Wiedmann, C. Bruene, A. Roth, H. Buhmann, L. W. Molenkamp, X.-L. Qi, and S.-C. Zhang, Science 318, 766 (2007).

2 L. Fu and C. L. Kane, Phys. Rev. Lett. 100, 096407 (2008).

3 C. Nayak, S. H. Simon, A. Stern, M. Freedman, and S. D. Sarma, Rev. Mod. Phys. 80, 1083 (2008).

4 D. A. Pesin and L. Balents (2009).

${ }^{5}$ C. Xu, Phys. Rev. B 81, 020411 (2010).

${ }^{6}$ C. Xu, Phys. Rev. B 81, 054403 (2010).

7 L. Fu, arXiv:0909.5172 (2009).

8 H.-J. Kwon, K. Sengupta, and V. M. Yakovenko, Low Temperature Physics 30, 613 (2004).

9 M. P. A. Fisher, P. B. Weichman, G. Grinstein, and D. S. Fisher, Phys. Rev. B 40, 546 (1989).

${ }^{10} \mathrm{~V}$. Shivamoggi and J. E. Moore (2009), URL http://meetings.aps.org/link/BAPS.2009.MAR.Q33.11.

11 V. Shivamoggi, G. Refael, and J. E. Moore, In preparation.

12 A. Kitaev, Annals Phys. 303, 2 (2003).

13 T. Senthil and M. P. A. Fisher, Phys. Rev. B 62, 7850 (2000).

14 P. Calabrese, A. Pelissetto, and E. Vicari, arXiv:cond- mat/0306273 (2003).

15 A. V. Chubukov, S. Sachdev, and T. Senthil, Nucl. Phys. B 426, 601 (1994).

16 C. Xu and S. Sachdev, Phys. Rev. B 79, 064405 (2009).

17 X.-G. Wen, Phys. Rev. Lett. 90, 016803 (2003).

18 C. Xu and J. E. Moore, Phys. Rev. Lett. 93, 047003 (2004).

19 J. Vidal, R. Thomale, K. P. Schmidt, and S. Dusuel, Phys. Rev. B 80, 081104(R) (2009).

20 R. Orus, A. C. Doherty, and G. Vidal, Phys. Rev. Lett. 102, 077203 (2009).

${ }^{21}$ C. Xu and J. E. Moore, Nucl. Phys. B 716, 487 (2004).

${ }^{22}$ H.-D. Chen, C. Fang, J. Hu, and H. Yao, Phys. Rev. B 75, 144401 (2007).

23 E. Fradkin and L. Susskind, Phys. Rev. D 17, 2637 (1978).

24 L. Balents and M. P. A. Fisher, Phys. Rev. B 71, 085119 (2005).

25 A. Paramekanti, L. Balents, and M. P. A. Fisher, Phys. Rev. B 66, 054526 (2002).

${ }^{26}$ C. $\mathrm{Xu}$ and L. Fu, to appear (2009). 